Abstract

\title{
Entropy Measurements with Infrared Sensors ${ }^{\dagger}$
}

\author{
Xavier Zamora and Angel Cuadras
}

Universitat Politècnica de Catalunya, BarcelonaTech, EEBE-DEEL, Terrassa, Spain

† Presented at the Entropy 2021: The Scientific Tool of the 21st Century, 5-7 May 2021; Available online: https://sciforum.net/conference/Entropy2021/.

Published: 5 May 2021

Infrared sensors (IRS) have been used for long to measure non-contact body temperatures, as in thermal cameras. These sensors are based on thermopiles that measure heat flows, which are converted to temperature through sensor's calibration curve. In this contribution we aim to combine the physical heat measurements with the inferred temperature measurements of the emitter to characterize the emitter radiated entropy reaching the sensor. We implemented a data acquisition system based on Arduino UNO microcontroller. Several IRS sensors, which had both thermopile and thermistor for independent environment temperature were tested. As thermal emitters, we used resistors whose power dissipation could be selected by controlling their current and voltage. We investigated the emitter transferred entropy reaching the IRS sensor as a function of emitter-sensor distance and the emitter dissipated power. Thus, the measurement system manages the emitter temperature and power dissipation, the heat reaching the sensor and the temperature of the sensor. From this data, we were able to monitor non-contact radiated entropy. This setup was used to characterize resistor ageing as resistor ageing is related to its entropy production. Degradation accelerated tests were carried out. Emitter resistors were submitted to dissipation powers well beyond their nominal power rate to speed up its degradation mechanisms. We were, thus, able to monitor resistor degradation with a non-contact sensor by evaluating the entropy reaching the sensor.

(C) 2021 by the authors. Licensee MDPI, Basel, Switzerland. This article is an open access article distributed under the terms and conditions of the Creative Commons Attribution (CC BY) license (http://creativecommons.org/licenses/by/4.0/). 